\title{
Star Formation in Self-Gravitating Molecular Cloud: The Critical Mass and the Core Accretion Rate
}

\author{
Gemechu M. Kumssa1,2,3, S. B. Tessema ${ }^{1,2}$ \\ ${ }^{1}$ Astronomy and Astrophysics Research Division, Entoto Observatory and Research Center (EORC), Ethiopian Space Science and \\ Technology Institute (ESSTI), Addis Ababa, Ethiopia \\ ${ }^{2}$ Addis Ababa University, Addis Ababa, Ethiopia \\ ${ }^{3}$ Department of Physics, College of Natural Sciences, Jimma University, Jimma, Ethiopia \\ Email: gemechumk@gmail.com, tessemabelay@gmail.com
}

How to cite this paper: Kumssa, G.M. and Tessema, S.B. (2020) Star Formation in Self-Gravitating Molecular Cloud: The Critical Mass and the Core Accretion Rate. World Journal of Mechanics, 10, 53-67. https://doi.org/10.4236/wjm.2020.105005

Received: April 24, 2020

Accepted: May 26, 2020

Published: May 29, 2020

Copyright $\odot 2020$ by author(s) and Scientific Research Publishing Inc. This work is licensed under the Creative Commons Attribution International License (CC BY 4.0).

http://creativecommons.org/licenses/by/4.0/

(c) (i) Open Access

\begin{abstract}
Understanding how stars form in molecular clouds is one of the ongoing research areas in astrophysics. Star formation is the fundamental process to which our current understanding remains incomplete due to the complexity of the physics that drives their formation within molecular clouds. In this article theoretical modelling of the lowest possible mass of the cloud needed for collapse and the core accretion rate has been presented for the molecular cloud collapsing under its gravity. In many of previous studies the critical mass of star forming cloud under its gravity has been modelled using kinetic energy and gravitational potential energy. However, we test the effect of thermodynamic efficiency factor together with other physical processes in describing the critical mass, and controlling or triggering the rate of mass falling onto the central core. Assuming that, the ratio of radiation luminosity to gravitational energy released per unit time of the collapsing MC is less than unity. Following this conceptual framework we have formulated the critical mass and the core accretion rate of the self-gravitating molecular cloud.
\end{abstract}

\section{Keywords}

Star Formation, Molecular Cloud, Critical Mass, Core Accretion

\section{Introduction}

The study of star formation provides important information to understand the process of planet formation and the evolution of galaxy. Since, the formation of stars and planets in molecular clouds is a crucial field of study in astronomy and 
astrophysics. As stated by [1] molecular cloud (MC) is the dense and cool region in interstellar medium (ISM), and its size assumed to be $\sim 0.1 \mathrm{pc}$ to $\sim 100 \mathrm{pc}$. It provides raw material for star and planet formation. However, how and why the cloud collapses to form star is another key question in astronomy. The boundaries of MCs are determined using the transitions from molecular gas like $\mathrm{H}_{2}$ to the atomic gas commonly $\mathrm{H}$ [2] [3]. The number of particles per unit volume (n) of the densest parts of MC is approximated to $\gtrsim 1000 \mathrm{~cm}^{-3}$ [4], temperature of this densest region is $T<100 \mathrm{~K}$. It is in this region where star formation is actively going on. Stars form over a wide mass range from $0.08 M_{\odot}$ up to $150 M_{\odot}$ and beyond (see [5] [6]). In our case, we mainly focus on low and intermediate star forming critical mass of the cloud and its core accretion rate.

As illustrated by [7] the majority of stars ( 90\%) are low mass stars with masses less than $0.8 M_{\odot}$, whereas about $10 \%$ are intermediate-mass stars have masses between 0.8 and $8 M_{\odot}$, where as stars with masses $\left(M_{\star}>8 M_{\odot}\right)$ are said to be massive stars, they are only about $1 \%$ of all stars. There is a big difference between massive star formation processes and low mass star formation processes. The peculiar property of high mass star formation is that the young star accretes mass even if it begun nuclear fusion. No one is entirely sure what the dominant and unavoidable pressure force in addition to gravity in contracting MCs to star is another critical issue in astrophysics. As [8] and [9] explained that supersonic turbulence will dissipate on the free-fall time scale, and magnetic pressure cannot stop the collapse [10] [11]. So that the concept of star formation in a cloud contracting by its own gravity needs additional understanding with its thermodynamic properties as we thought.

According to [12] [13] [14] cold MCs have been heated by the photons emitted by high mass stars and inhibit the collapse of a cloud. On the other hand, [15] [16] explained super nova (SN) explosions and the HII region expansion collect the gas together and trigger star formation in galaxies. The effects of these feedbacks are important in regulating star formation in galaxies [17]. What if no external agents, the role of gravity in SF? The central core accretion rate is in the early collapse phase of MC. Thus, in this study we mainly focus on formulating another alternative of critical mass and the core accretion rate.

As described by [1] pre-stellar core is the first collapsed region of the star forming MC. In the low star forming region the pre-stellar core mass is from $\sim 0.5 M_{\odot}$ to $10 M_{\odot}$, but the hydrostatic object found at the center of the core is termed as protostar. The hydrostatic core grows to star via accretion of the material onto the central core from the envelope. Moreover, how the central core accretion rate can theoretically be explained is a crucial to theoretical astrophysics, thus this paper aims to formulate equation of the core accretion based on the critical mass we modelled in our earlier paper [18].

On the other way, the mass infall rate of the central core has not yet been theoretically modelled based on the parent cloud property including thermodynamic efficiency factor. Therefore, we model equation of core accretion rate of 
the central core or very young protostar which is embedded in the center of initially collapsing MC or pre-stellar core. The purpose of the study is: formulating critical mass (Jeans mass) of self-gravitating molecular cloud and mathematically formulating core accretion rate. Describe the hierarchy from fragmentation to star formation through invoking an efficiency factor $\epsilon$ into equation of radiation luminosity. The general method we apply is theoretical modelling followed by numerical calculation.

\section{Review of the Jeans Mass \& Bonnor-Ebert Mass Scale}

The jeans mass scale and the Bonner-Ebert mass scale are the classic mass scales for the contraction of a gravitating MC. Jeans mass: is Minimum or critical mass that is necessary to initiate the spontaneous collapse of the cloud. We believe that stars form when a portion of a molecular cloud collapses gravitationally. Since this collapse is resisted by various things, and there is evidence for many molecular clouds that have not collapsed, it is clear that the collapse initiating star formation occurs only under some circumstances. Can we find a simple condition that tells us when a cloud becomes unstable to collapse? This question was answered in a very simple model by James Jeans, who showed that for a cloud of a given radius and temperature, there is a critical mass that is now called the Jeans mass. If it is exceeded, the cloud becomes unstable to collapse. The Jeans mass depends on the radius of the cloud, its temperature, and the average mass of the particles in the cloud. The Jeans mass is determined by asking when the magnitude of the gravitational potential energy exceeds the magnitude of the gas kinetic energy. It is given by $M_{J}=\left(\frac{5 K T_{c}}{G \mu m_{H}}\right)^{3 / 2}\left(\frac{3}{4 \pi \rho_{c}}\right)^{1 / 2}$ where $k$ is the Boltzmann constant, $T_{c}$ the temperature, $\rho_{c}$ the density of the cloud, $R_{c}$ the radius of the cloud, $G$ the gravitational constant, and $\mu m_{H}=m$ the average mass of a gas particle. If the cloud's mass exceeds this value, gravity can overcome the gas kinetic energy and initiate collapse.

Bonner-Ebert Mass Scale: is the maximum mass for an isothermal sphere embedded in a pressurized medium in hydrostatic equilibrium (in the absence of magnetic fields and turbulence), the Bonnor-Ebert mass is given by $M_{B E}=1.182 \frac{\sigma_{t h}^{3}}{\left(G^{3} \rho_{o}\right)^{1 / 2}}=1.182 \frac{\sigma_{t h}^{4}}{\left(G^{3} P_{t h, o}\right)^{1 / 2}}$ where $P_{t h, o}=\rho_{o} \sigma^{2}$ is the surface pressure (Bonnor, 1956; Ebert, 1957). Clouds with $M_{c}>M_{B E}$ cannot be prevented from collapsing by thermal pressure alone.

Magnetic Filed Mass Scale: Magnetic fields alone cannot prevent gravitational collapse in clouds with $M_{c}>M_{\phi}$ is given by $M_{\Phi}=C_{\Phi} \frac{\Phi}{G^{1 / 2}}$ where $\Phi$ is the magnetic flux in the cloud, and $C_{\Phi}$ is a constant which depends on the configuration of the magnetic field. Clouds with $M_{c}>M_{\Phi}$ and $M_{c}<M_{\Phi}$ are said to be magnetically supercritical and subcritical respectively. Apart from these classical models, in this paper, we formulate the critical mass with respect to ra- 
dius, temperature and the ratio of radiation luminosity to gravitational luminosity of the collapsing cloud through reviewing our published paper (i.e. [18]). In addition we model core accretion rate in two ways.

\section{Basic Equations and Concepts}

In this section we describe gravitational potential energy of a spherical cloud, the released energy during collapse, gravitational energy released per free-fall time, and setting assumptions to derive relation between gravitational luminosity and radiation luminosity.

\subsection{Gravitational Potential Energy of a Spherical Cloud}

The total gravitational potential (binding) energy of a sphere cloud of uniform density is given by

$$
E_{g}=-\int_{0}^{M_{c}} \frac{G M(r)}{r} \mathrm{~d} m
$$

where $M(r)$ is the mass contained in radius $r$. The gravitational potential energy of a system is the energy required to assemble the mass by bringing matter from infinity to the point of interest. Substituting $\mathrm{d} m=4 \pi \rho_{c} r^{2} \mathrm{~d} r$, and $\rho_{c}=\frac{3 M_{c}}{4 \pi R_{c}^{3}}$ Equation (1) becomes

$$
E_{g}=-\int_{0}^{R} \frac{G\left(\frac{4}{3} \pi \rho r^{3}\right)}{r} 4 \pi \rho r^{2} \mathrm{~d} r=-\int_{0}^{R} \frac{16}{3} G \pi^{2} \rho^{2} r^{4} \pi r
$$

Substituting $\rho$ we have

$$
E_{g}=-\frac{16}{15} G \pi^{2} \rho^{2} R^{5}=-\frac{3 G M^{2}}{5 R}
$$

Therefore, a spherical cloud with mass $M_{c}$, density $\rho_{c}$ and radius $R_{c}$ has gravitational energy given by

$$
E_{g}=-c \frac{G M_{c}^{2}}{R_{c}}
$$

where $c$ is a constant depending on the cloud density. We choose the value of this constant either $3 / 5$ or 1 when we assume spherical uniform density cloud or need to consider uncertainty respectively [18]. From the Virial theorem $E_{g}+2 U=E_{g}+2 E_{k}=0$, this indicates that gravitational energy and the internal energy are in balance means the MCs neither collapse nor expand. The total energy of the system is related to gravitational potential energy $E_{g}$ by $E_{\text {tot }}=E_{g}+U=E_{g}-\frac{1}{2} E_{g}=\frac{1}{2} E_{g}$, it implies that the internal energy must increase as the gravitational energy goes to negative. The total energy $\left(E_{\text {tot }}\right)$ is negative means the molecular cloud is bound and stable. Which must be negative as cloud is bound (i.e. $E_{g}<0$ ), gravitational systems tend to do this very rapidly, a bound system that is not in virial equilibrium will change its configuration very 
rapidly to get into virial equilibrium.

This idea rests on the fact that the gravitational energy $E_{g}$ has a negative sign, and it is a confining agent, while the internal energies (thermal, kinetic, or magnetic) are positive, and they are assumed to act as supporting agents against collapse. It is frequently described in the literature that if $E_{g}+2 U=E_{g}+2 E_{k}<0$, the cloud must be contracting, while $E_{g}+2 U=E_{g}+2 E_{k}>0$ implies that the cloud is expanding. For the MC to collapse $\left|\frac{E_{g}}{2}\right|>E_{k}$.

\subsection{The Time Scale}

The characteristic time scale associated with contraction of self-gravitating molecular cloud is the free-fall time. The time scale of star formation under a self-gravitating cloud is often estimated by this time scale or the time it would take a cloud to collapse to a point in the absence of any other forces. By neglecting pressure gradients, the free fall time for spherically symmetric homogeneous collapsing cloud with mass $M_{c}$ and radius $R_{c}$ is given by

$$
t_{f f}=\left[\frac{3 \pi}{32 G \rho_{c}}\right]^{\frac{1}{2}}
$$

where $\rho_{c}$ is the initial density of the molecular cloud under consideration.

\subsection{Assumptions and Approximations}

We choose the value of $\mathrm{c}$ in Equation (3) is $3 / 5$ for a uniform density assumption and 1 otherwise. However, the density profile of the molecular cloud is more likely to be centrally peaked, so we suggested using a numerical factor of order unity to express the small uncertainty. When a cloud core is collapsing under its own gravity, half of its total gravitational energy is radiated away and half goes to internal energy according to the Virial theorem. However, we assume that the amount of gravitational energy released during the collapse is not completely (100\%) liberated away in the form of radiation. Therefore, some part of the released energy may be trapped by other cosmic matter at the boundary without being involved in changing temperature of the collapsing cloud core. Due to this reason we impose a dimensionless thermodynamic factor $\epsilon$ which we quantify it as

$$
\epsilon=\frac{L_{\text {rad }}}{L_{g f}}
$$

where $L_{\text {rad }}$ is the radiation luminosity of the collapsing cloud at its effective temperature and $L_{g f}$ is the gravitational luminosity in free-fall time.

\subsection{The Released Energy during Collapse}

The gravitational energy released when a cloud of radius $R_{c}$ collapsed to the surface of a pre-stellar of radius $R_{\text {core }}$ (see [18]) is given by 


$$
\begin{gathered}
\Delta E_{g}=\left[\frac{G M_{c}^{2}}{R_{\text {core }}}-\frac{G M_{c}^{2}}{R_{c}}\right] \\
\Delta E_{g}=G M_{c}^{2}\left[\frac{1}{R_{\text {core }}}-\frac{1}{R_{c}}\right] \\
\Delta E_{g}=\frac{G M_{c}^{2}}{R_{\text {core }}}
\end{gathered}
$$

Here we assumed $\frac{1}{R_{c}} \ll$. This implies the energy released is mainly dependent on the core radius, the radius of the core. However, when we consider the total gravitational energy released by the cloud of mass $M_{c}$ is more sensitive to the parent cloud radius the gravitational energy is expressed as

$\Delta E_{g}=G M_{c}^{2}\left[\frac{1}{R_{c}}-\frac{1}{R_{\infty}}\right] \approx \frac{G M_{c}^{2}}{R_{c}}$, therefore, we set out two different mathematical frameworks. Half of the energy described in Equation (8) is liberated according to the Virial theorem, thus we have

$$
E_{\text {grad }}=\frac{1}{2} \Delta E_{g}=\frac{G M_{c}^{2}}{2 R_{\text {core }}}
$$

$E_{\text {grad }}$ is read as the gravitational energy radiated away.

\subsection{Gravitational Energy Released per Free-Fall Time}

To undergo free fall the gravitational energy of the cloud has to dominate other forms of energy pressures opposing it. Remembering that the gravitational pressure is dominating the counteracting pressures and the $\mathrm{MC}$ is falling to the central region. Now we can calculate the luminosity due to gravity in-terms of the free fall time as

$$
L_{g f}=\frac{\text { gravitational energy radiated away }}{\text { freefall time }}=\frac{E_{\text {grad }}}{t_{f f}}
$$

Thus using $t_{f f}$ in Equation (4), Equation (8) and Equation (10) we have

$$
\begin{aligned}
L_{g f} & =\frac{G M_{c}^{2}}{2 R_{\text {core }}}\left(\frac{32 G \rho_{c}}{3 \pi}\right)^{\frac{1}{2}}=\frac{G^{3 / 2} M_{c}^{2}}{2 R_{\text {core }}}\left(\frac{32 \rho_{c}}{3 \pi}\right)^{1 / 2} \\
& =1.8426 \frac{G^{3 / 2} M_{c}^{2}}{2 R_{\text {core }}}\left(\frac{3 M}{4 \pi R^{3}}\right)^{1 / 2} \\
& =0.9003 \frac{G^{3 / 2} M_{c}^{2}}{2 R_{\text {core }}}\left(\frac{M_{c}}{R_{c}^{3}}\right)^{1 / 2}
\end{aligned}
$$

After substituting the value of $G$ and doing little manipulation $L_{g f}$ is formulated by [18] ad is given by

$$
L_{g f} \approx 3.0721 \times 10^{-17}\left(\frac{M_{c}^{5}}{R_{c}^{3} R_{\text {core }}^{2}}\right)^{\frac{1}{2}} \mathrm{~J} / \mathrm{s}
$$

where $M_{c}$ and $R_{c}$ are the mass and radius of the parent cloud respectively, $L_{g f}$ 
gravitational luminosity and $t_{f f}=\left[\frac{3 \pi}{32 G \rho_{c}}\right]^{\frac{1}{2}}, \quad G \approx 6.67 \times 10^{-11} \mathrm{~m}^{3} \cdot \mathrm{kg}^{-1} \cdot \mathrm{s}^{-2}$ $\left(6.67 \times 10^{-8} \mathrm{~cm}^{3} \cdot \mathrm{g}^{-1} \cdot \mathrm{s}^{-2}\right)$ is $\rho_{c}=\frac{3 M}{4 \pi R^{3}}$ denotes the initial density of the cloud. From the general gravitational energy released Equation (11) becomes $L_{g f} \approx 3.0721 \times 10^{-17}\left(\frac{M_{c}^{5}}{R_{c}^{5}}\right)^{\frac{1}{2}} \mathrm{~J} / \mathrm{s}$. Note that we have been using $R_{c}$ for the parent cloud radius before fragmented into cores, and $R_{\text {core }}$ is the radius of the core after initial cloud fragmented or collapsed to pre-stellar core. If cloud cools enough, Jeans instability allows gravity to overtake thermal energy. The densest parts of the cloud become gravitationally unstable and fragmentation occurs. It is after this fragmentation $R_{\text {core }}$ has been assumed. For small initial cloud with very small Jeans mass the core radius becomes the radius of the pre-stellar core formed from the rapid collapse of the initial cloud. Thus there is a clear difference between $R_{c}$ and $R_{\text {core }}$ in this paper.

\section{Results}

\subsection{Theoretical Modelling of Critical Mass and Core Accretion Rate}

In this section we adopt the assumption and procedures which have been used in our previous paper (see [18]), and describe $\epsilon$ as well as to obtain the initial mass of a cloud, thus

$$
\frac{4 \pi R_{c o r e}^{2} \sigma T_{c}^{4}}{L_{g f}}=\epsilon
$$

and remodel critical mass in two different ways to avoid some ambiguity. Where $L_{\text {rad }}=4 \pi R_{\text {core }}^{2} \sigma T_{c}^{4}, \sigma$ is Stefan-Boltzmann's constant, $T_{c}$ is the cloud's temperature. Then from Equation (13) we have

$$
4 \pi R_{\text {core }}^{2} \sigma T_{c}^{4}=\epsilon \times 3.0721 \times 10^{-17}\left(\frac{M_{c}^{5}}{R_{c}^{3} R_{\text {core }}^{2}}\right)^{\frac{1}{2}}
$$

implies

$$
\begin{gathered}
M_{c}^{5 / 2}=\frac{4 \pi R_{\text {core }}^{2} \sigma T_{c}^{4} R_{c}^{3 / 2} R_{\text {core }}}{\epsilon \times 3.0721 \times 10^{-17}} \\
M_{c}=\left(\frac{4 \pi \sigma}{3.0721 \times 10^{-17}}\right)^{2 / 5}\left(\frac{R_{\text {core }}^{3} T_{c}^{4} R_{c}^{3 / 2}}{\epsilon}\right)^{2 / 5} \\
M_{\text {crt }} \approx 1.40 \times 10^{4}\left(\frac{R_{c}^{3} R_{\text {core }}^{6} T_{c}^{8}}{\epsilon^{2}}\right)^{\frac{1}{5}}[\mathrm{~kg}] \approx 1.40 \times 10^{4}\left(\frac{R_{c}^{3} R_{\text {core }}^{6} T_{c}^{8}}{\epsilon^{2}}\right)^{\frac{1}{5}}\left[\frac{M_{\odot}}{2 \times 10^{30}}\right] \\
\approx 7.00 \times 10^{-27}\left(\frac{R_{c}^{3} R_{\text {core }}^{6} T_{c}^{8}}{\epsilon^{2}}\right)^{\frac{1}{5}}\left[M_{\odot}\right]
\end{gathered}
$$


When we turn to the general method i.e. if the gravitational energy liberated is more influenced by the parent cloud radius we have

$M_{c r t} \approx 1.40 \times 10^{4}\left(\frac{R_{c}^{9} T_{c}^{8}}{\epsilon^{2}}\right)^{\frac{1}{5}}\left[\frac{M_{\odot}}{2 \times 10^{30}}\right]$. Therefore, we set two solutions of the cloud mass as

$$
M_{c r t}= \begin{cases}7.00 \times 10^{-27}\left(\frac{R_{c}^{3} R_{c o r e}^{6} T_{c}^{8}}{\epsilon^{2}}\right)^{\frac{1}{5}}\left[M_{\odot}\right], & \text { if sensitive to } R_{\text {core }} \cdot \quad \text { (a) } \\ 7.00 \times 10^{-27}\left(\frac{R_{c}^{9} T_{c}^{8}}{\epsilon^{2}}\right)^{\frac{1}{5}}\left[M_{\odot}\right], & \text { otherwise. }\end{cases}
$$

where $\sigma=5.67 \times 10^{-8} \mathrm{~J} \cdot \mathrm{s}^{-1} \cdot \mathrm{m}^{-2} \cdot \mathrm{K}^{-4}$, As the rate of releasing gravitational energy per unit time is increasing the minimum mass required for collapse reduces. On the other hand, where there is no release of gravitational energy there is no collapse. Equation (16) shows large critical mass has large radius, and the smaller the radius of the cloud the smaller is its critical mass. Hence we considered $\rho_{c}=\frac{M_{c}}{4 / 3 \pi R_{c}^{3}}$, then from Equation (15).

$$
\begin{aligned}
& \frac{4 \pi R_{c}^{3} \rho_{c}}{3}=\left(1.40 \times 10^{4}\right)\left(\frac{R_{c}^{3} R_{c o r e}^{6} T_{c}^{8}}{\epsilon^{2}}\right)^{\frac{1}{5}} \\
& \rho_{c}=\left(1.40 \times 10^{4}\right) \frac{3}{4 \pi R_{c}^{3}}\left(\frac{R_{c}^{3} R_{\text {core }}^{6} T_{c}^{8}}{\epsilon^{2}}\right)^{\frac{1}{5}}
\end{aligned}
$$

Thus

$$
\rho_{c r t} \approx 3.342 \times 10^{3}\left[\frac{R_{c o r e}^{6} T_{c}^{8}}{\epsilon^{2} R_{c}^{12}}\right]^{1 / 5} \mathrm{~kg} \cdot \mathrm{m}^{-3}
$$

The radius from Equation (15) is obtained as

$$
R_{c}^{3 / 5}=\frac{M_{c} \epsilon^{2 / 5}}{\left(1.40 \times 10^{4}\right) R_{\text {core }}^{6 / 5} T_{c}^{8 / 5}}
$$

leads to

$$
R_{c}=\frac{M_{c}^{5 / 3} \epsilon^{2 / 3}}{\left(1.40 \times 10^{4}\right)^{5 / 3} R_{\text {core }}^{2} T_{c}^{8 / 3}}
$$

rearranging this we have

$$
R_{c r t} \approx 1.2297 \times 10^{-7}\left[\frac{\epsilon^{2} M_{c}^{5}}{R_{\text {core }}^{6} T_{c}^{8}}\right]^{\frac{1}{3}} \mathrm{pc}
$$

From Equation (18)

$$
T_{c}=\frac{\epsilon^{1 / 4} M_{c}^{5 / 8}}{\left(1.40 \times 10^{4}\right)^{5 / 8} R_{\text {core }}^{6 / 8} R_{c}^{3 / 8}}
$$


temperature of the critical mass is

$$
T_{c} \approx 0.0026\left[\frac{\epsilon^{2} M_{c}^{5}}{R_{c}^{3} R_{\text {core }}^{6}}\right]^{\frac{1}{8}} \mathrm{~K}
$$

How the thermodynamic efficiency factor is governing the critical mass of self-gravitating cloud is displayed in Figure 1. Figures 1-4 indicate the total critical mass contained in the radii with the other physical parameters addressed in their captions. Since the Jeans mass is a minimum mass, the unstable collapsing cloud could begin with a very large mass, may be even thousands of solar masses. So why, don't we get such massive stars? There are a lot of reasons, but one is that as the density increases hugely, the temperature becomes very low, the radius reduces rapidly, and as the ratio of radiation to gravitational energy increases. Thus the Jeans mass becomes smaller, and so large clouds will fragment. Final fragments will tend to be on the order of a solar mass. Thus, an initial massive collapsing cloud will fragment into many small collapsing protostars lead star cluster. For the diffuse HI cloud the critical mass is about thousands of solar mass, whereas molecular cloud core has critical mass of solar mass scale.

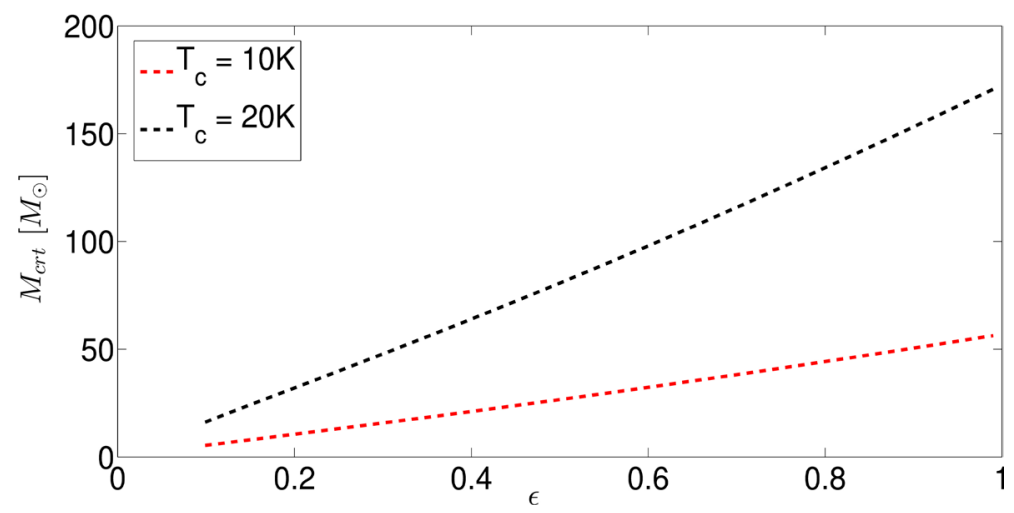

Figure 1. Luminosity ratio $(\epsilon)$ vs critical mass. When a cloud of radius $R_{c} \sim 1 \mathrm{pc}-1.99 \mathrm{pc}$ collapsed to $R_{\text {core }} \sim 0.0001 \mathrm{pc}$ to $0.00109 \mathrm{pc}$ in the run with indicated temperatures in the plot The plot is obtained from Equation (15).

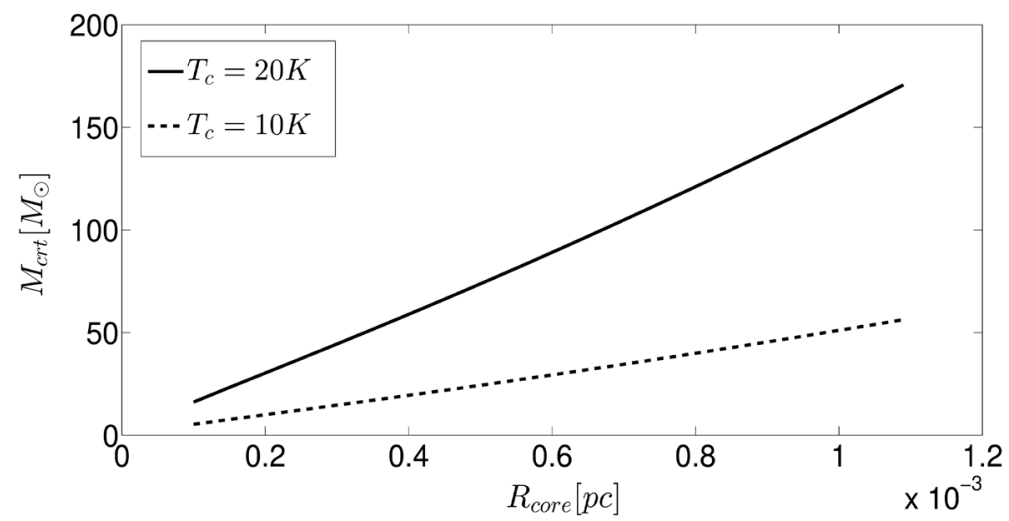

Figure 2. The formed core radius $(\epsilon)$ vs critical mass of the initial cloud. When a cloud of radius $R_{c} \sim 1 \mathrm{pc}-1.99 \mathrm{pc}$ collapsed to $R_{\text {core }} \sim 0.0001 \mathrm{pc}$ to $0.00109 \mathrm{pc}$ with indicated temperatures in the plot and $\epsilon=0.099-0.99$. The plot is obtained from Equation (15). 


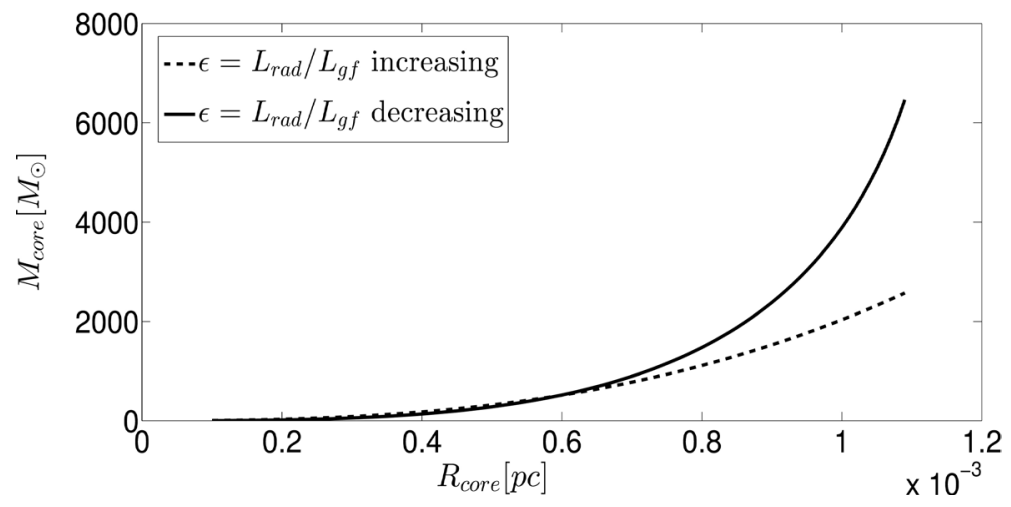

Figure 3. Radius of the newly formed core vs critical mass of the initial cloud. When the initial cloud radius $R_{c} \sim$ runs from $1 \mathrm{pc}-1.99 \mathrm{pc}$ collapsed to $R_{\text {core }}$ indicated in the plot, with running temperature $T_{c} \sim 10-109 \mathrm{~K}$ and $\epsilon=0.099-0.99$ for increasing. The plot is obtained from Equation (15).

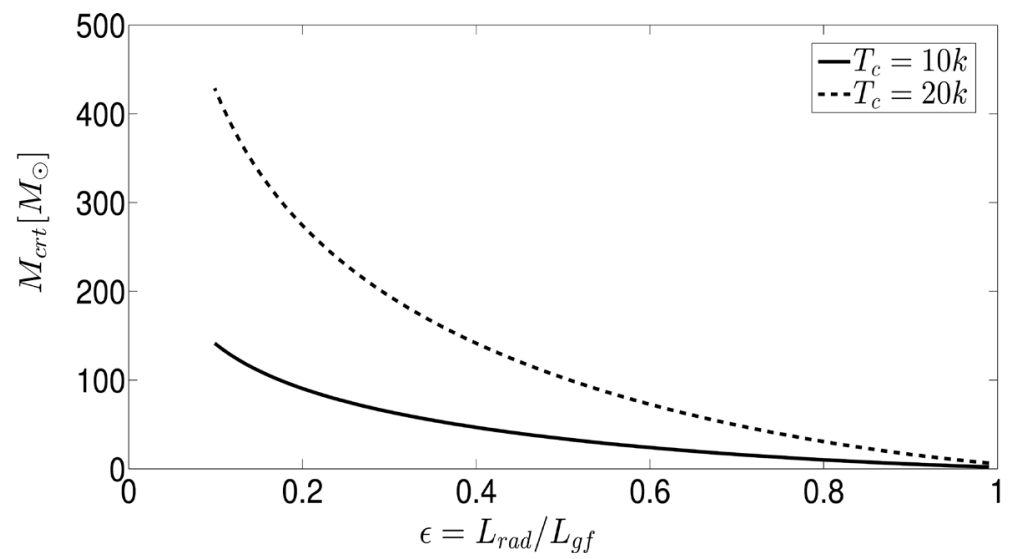

Figure 4. Cloud's critical mass vs the ratio of its luminosities. When the initial cloud radius $R_{c} \sim$ runs from $1 \mathrm{pc}-1.99 \mathrm{pc}$ collapsed to $R_{\text {core }}$ indicated in the plot. When we see curves in this figure thermodynamic efficiency factor plays grate role in describing critical mass.

\subsection{The Core Accretion Rate}

It is the rate at which the central object (central core) gains mass from the initially collapsing cloud. This mass infall rate depends on the properties of the minimum mass of the cloud required for collapse. When the cloud is collapsing the mass is falling onto the central core. This process depends on time and properties of the cloud. Thus keeping $\epsilon$ constant and considering spherical accretion. Since for a roughly spherical gas cloud with a mass close to the Jeans mass we can estimate the average accretion rate by taking the derivative of the critical mass with respect to time as:

$$
\dot{M}_{\text {core }}=\frac{1.40 \times 10^{4}}{\epsilon^{2 / 5}} \times\left[\frac{3 R_{c}^{2} R_{\text {core }}^{6} T_{c}^{8} \dot{R}_{c}+6 R_{c}^{3} R_{\text {core }}^{5} T_{c}^{8} \dot{R}_{\text {core }}+8 R_{c}^{3} R_{\text {core }}^{6} T_{c}^{7} \dot{T}_{c}}{5\left(R_{c}^{3} R_{\text {core }}^{6} T_{c}^{8}\right)^{4 / 5}}\right] \mathrm{kg} \cdot \mathrm{s}^{-1}
$$

Hence $1 M_{\odot} \mathrm{yr}^{-1}=6.3412 \times 10^{22} \mathrm{~kg} \cdot \mathrm{s}^{-1}$, then Equation (20) in $M_{\odot} \mathrm{yr}^{-1}$ can be expressed as 


$$
\dot{M}_{\text {core }}=\frac{1.4 \times 10^{4}}{6.3412 \times 10^{22} \epsilon^{2 / 5}} \times\left[\frac{3 R_{c}^{2} R_{\text {core }}^{6} T_{c}^{8} \dot{R}_{c}+6 R_{c}^{3} R_{\text {core }}^{5} T_{c}^{8} \dot{R}_{\text {core }}+8 R_{c}^{3} R_{\text {core }}^{6} T_{c}^{7} \dot{T}_{c}}{5\left(R_{c}^{3} R_{\text {core }}^{6} T_{c}^{8}\right)^{4 / 5}}\right]
$$

Simplifying and using all conversion parameters the mass infall rate for the core radius dominating cases is

$$
\dot{M}_{\text {core }} \approx \frac{2.21 \times 10^{-19}}{\epsilon^{2 / 5}} \times\left[\frac{3 R_{c}^{2} R_{\text {core }}^{6} T_{c}^{8} \dot{R}_{c}+6 R_{c}^{3} R_{\text {core }}^{5} T_{c}^{8} \dot{R}_{\text {core }}+8 R_{c}^{3} R_{\text {core }}^{6} T_{c}^{7} \dot{T}_{c}}{5\left(R_{c}^{3} R_{\text {core }}^{6} T_{c}^{8}\right)^{4 / 5}}\right]\left[M_{\odot} \mathrm{yr}^{-1}\right]
$$

Of course temperature cannot remain constant throughout the collapse process. In this case, we have a much more means to find the accretion rate; so that to simplify the changing temperature in time we describe as $\dot{T}_{c}=\frac{\mathrm{d} T_{c}}{\mathrm{~d} t}=\frac{\mathrm{d} T_{c}}{\mathrm{~d} t} \frac{\mathrm{d} R_{\text {core }}}{\mathrm{d} R_{\text {core }}}=\dot{R}_{\text {core }} \frac{\mathrm{d} T_{c}}{\mathrm{~d} R_{\text {core }}}=v_{\text {(core) }} \frac{\mathrm{d} T_{c}}{\mathrm{~d} R_{\text {core }}}$, where $\frac{\mathrm{d} T_{c}}{\mathrm{~d} R_{\text {core }}} \quad$ is approximated to $\frac{\delta T_{c}}{\delta R_{\text {core }}}$. Where $\dot{R}_{c}=v_{(c)}$ and $\dot{R}_{\text {core }}=v_{\text {(core) }}$ is the radial infall velocity of matter in the core and parent cloud respectively. Thereafter we Let $\chi=\frac{2.21 \times 10^{-19}}{\epsilon^{2 / 5}}$ and have $\dot{M}_{\text {core }} \approx \chi\left[\frac{3 R_{c}^{2} R_{\text {core }}^{6} T_{c}^{8} \dot{R}_{c}+6 R_{c}^{3} R_{\text {core }}^{5} T_{c}^{8} \dot{R}_{\text {core }}+8 R_{c}^{3} R_{\text {core }}^{6} T_{c}^{7} \dot{R}_{\text {core }} \frac{\delta T_{c}}{\delta R_{\text {core }}}}{5\left(R_{c}^{3} R_{\text {core }}^{6} T_{c}^{8}\right)^{4 / 5}}\right]\left[M_{\odot} \mathrm{yr}^{-1}\right]$

simplifying this

$$
\dot{M}_{\text {core }} \approx \chi\left[\frac{3 R_{c}^{2} R_{\text {core }}^{6} T_{c}^{8} \dot{R}_{c}+2 R_{c}^{3} R_{\text {core }}^{5} T_{c}^{7} \dot{R}_{\text {core }}\left(3 T_{c}+4 R_{\text {core }} \frac{\delta T_{c}}{\delta R_{\text {core }}}\right)}{5\left(R_{c}^{3} R_{\text {core }}^{6} T_{c}^{8}\right)^{4 / 5}}\right]\left[M_{\odot} \mathrm{yr}^{-1}\right]
$$

where $\chi$ is made constant for simplicity by making $\epsilon$ constant, otherwise changing. Equations (20) to (23) are when radius of the core plays very great role in describing the collapsing cloud mass. Moreover, this expression can also be simplified, in the case that the collapsing cloud is more sensitive to the initial cloud radius. Thus from Equation (16b) we have $\dot{M}_{\text {core }}=1.40 \times 10^{4} \frac{\mathrm{d}}{\mathrm{d} t}\left[\left(\frac{R_{c}^{9} T_{c}^{8}}{\epsilon^{2}}\right)^{\frac{1}{5}}\right]$. Assuming $\epsilon$ as constant in the general sense we have

$$
\dot{M}_{\text {core }} \approx \chi\left[\frac{9 R_{c}^{8} T_{c}^{8} \dot{R}_{c}+8 R_{c}^{9} T_{c}^{7} \dot{R}_{c} \frac{\delta T_{c}}{\delta R_{\text {core }}}}{5\left(R_{c}^{9} T_{c}^{8}\right)^{4 / 5}}\right]\left[M_{\odot} \mathrm{yr}^{-1}\right]
$$

So we have two equations of core accretion from Equations (23) \& (24) collectively written as 


$$
\dot{M}_{\text {core }} \approx\left\{\begin{array}{l}
\chi\left[\frac{3 R_{c}^{2} R_{\text {core }}^{6} T_{c}^{8} \dot{R}_{c}+2 R_{c}^{3} R_{\text {core }}^{5} T_{c}^{7} \dot{R}_{\text {core }}\left(3 T_{c}+4 R_{\text {core }} \frac{\delta T_{c}}{\delta R_{\text {core }}}\right)}{5\left(R_{c}^{3} R_{\text {core }}^{6} T_{c}^{8}\right)^{4 / 5}}\right]\left[M_{\odot} \mathrm{yr}^{-1}\right] . \text { (a) } \\
\chi\left[\frac{9 R_{c}^{8} T_{c}^{8} \dot{R}_{c}+8 R_{c}^{9} T_{c}^{7} \dot{R}_{c} \frac{\delta T_{c}}{\delta R_{\text {core }}}}{5\left(R_{c}^{9} T_{c}^{8}\right)^{4 / 5}}\right]\left[M_{\odot} \mathrm{yr}^{-1}\right] . \text { (b) }
\end{array}\right.
$$

We apply Equation (25a) if the effect of the core radius is more dominating than that of the parent MC and one can use Equation (25b) for the dominant case of the parent cloud radius. In this chapter we focus on the core dominating case.

\subsection{Numerical Calculation of Mass Infall Rate}

We assume $R_{c}$ is different from $R_{\text {core }}$ and the life of the initial cloud and the core also different then we set $t_{c}$ and $t_{c o r e}$ for the parent cloud and the core respectively. In this particular condition $t_{c}$ runs from $10^{7}-10^{8.04} \mathrm{yr}, t_{c o r e}$ runs from $10^{5}-10^{7} \mathrm{yr}$, thus $\dot{R}_{c}=R_{c} / t_{c}$, and $\stackrel{R}{c o r e}_{c}=R_{\text {core }} / t_{\text {core }}$. Moreover, assuming isothermal collapse and keeping $T_{c}=10 \mathrm{~K}$ and $\epsilon=0.9$. The initial cloud having radius $R_{c}=0.01 \mathrm{pc}$ collapsed in time to the core with reducing radius from $0.00001 \mathrm{pc}-0.0000001 \mathrm{pc}$ we plotted the figure below.

Figure 5 shows the accretion rate of the central core is governed by the rate of collapse of the initial cloud, the change of the central core radius in time, free-fall time and the size of the core. As the free-fall time of the cloud increases the rate of mass in fall reduces in time. This implies that matter in the reservoir gets depleted as free-fall time becomes so long. In general the figure indicates the
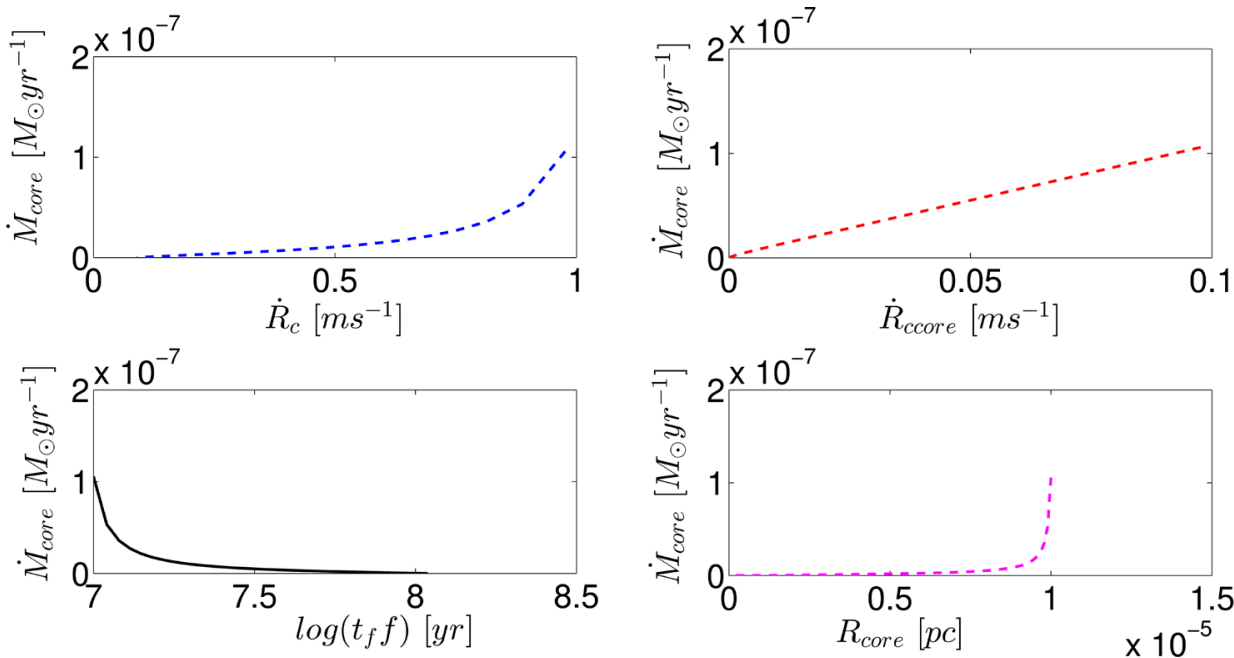

Figure 5. Numerically calculated core accretion rate with respect to different parameters. The plot is obtained from Equation (20). In general the curves of this figure show us how the radial flow supports the growth of acceptor. The rate of mass falling onto the central object reduces as time goes. 
mass infall rate is not only a matter of the physical properties of the cloud but also the life time.

\section{Summary}

Current star formation models are successful in explaining a wide variety of observed data, but remain incomplete. The star formation process in sgMC needs to be discussed within the context of the thermodynamic efficiency factor in addition to the initial properties (mass, density, radius, and temperature) of the parent cloud. From the results obtained and model, the following key conclusions could be drawn: There is no one and an all-time feasible method of calculating critical mass of star-forming $\mathrm{MC}$; methods vary according to the dynamical process that involved in collapsing the cloud core. Identification of the properties of the star-forming environment is the priorities as a key precondition for determining the fundamental parameters in collapsing a cloud and forming the star. Thermodynamic efficiency factor of self-gravitating MC is an additional constraint in studying star formation in sgMC. Due to these reasons it cannot be ignored in describing the critical mass of the sgMC.

The rate of mass infall onto the central core has been modelled in two ways, one when the accretion rate is more affected by the radius of the central star forming core, and two if the core accretion rate is more sensitive to the parent cloud's radius. In both cases we have shown how the amount of matter from the parent cloud or envelope falling onto the star forming core reduces in time. Since the cloud life time is lower than the cloud free-fall time there will be no supply of matter to the central core from the envelope. Therefore, both free-fall time and the parent cloud life time, and other dynamical parameters play the crucial role in describing core growth rate as well as star formation rate. The method somehow is adequate to address the gap in studying star formation.

\section{Acknowledgements}

We thank Ethiopian Space Science and Technology Institute (ESSTI), Entoto Observatory and Research Center (EORC), Department of Astronomy and Astrophysics for supporting this study. The International Science Programme (ISP)-Uppsala University and the East African Astrophysics Research Network (EAARN) are gratefully acknowledged for supporting this study. Our thanks also go to the anonymous reviewer. G.M. Kumssa, thanks Jimma University for supporting this study.

\section{Conflicts of Interest}

The authors declare no conflicts of interest regarding the publication of this paper.

\section{References}

[1] Ward-Thompson, D. and Whitworth, A.P. (2011) An Introduction to Star Forma- 
tion. Cambridge University Press, Cambridge.

[2] Blitz, L. (1988) Large Molecular-Cloud Complexes. In: Wolsten, R.D., Ed., Millimetre and Submillimetre Astronomy, Springer, Berlin, 269-280.

https://doi.org/10.1007/978-94-009-3019-3_10

[3] Blitz, L. (1991) Star Forming Giant Molecular Clouds. In: Lada, C.J. and Kylafis, N.D., Eds., The Physics of Star Formation and Early Stellar Evolution, Kluwer, Dordrecht, 3-34. https://doi.org/10.1007/978-94-011-3642-6_1

[4] Bertram, et al. (2016) Synthetic Observations of Molecular Clouds in a Galactic Centre Environment I. Studying Maps of Column Density and Integrated Intensity. MNRAS, 455, 3763-3778. https://doi.org/10.1093/mnras/stv2619

[5] Burrows, A., Hubbard, W.B., Lunine, J.I. and Liebert, J. (2001) The Theory of Brown Dwarfs and Extrasolar Giant Planets. Reviews of Modern Physics, 73, 719-765. https://doi.org/10.1103/RevModPhys.73.719

[6] Crowther, P.A., et al. (2010) The R136 Star Cluster Hosts Several Stars Whose Individual Masses Greatly Exceed the Accepted $150 \mathrm{M}_{\text {solar }}$ Stellar Mass Limit. MNRAS, 408, 731-751.

[7] Kroupa, P. (2002) The Initial Mass Function of Stars: Evidence for Uniformity in Variable Systems. Science, 295, 82-91. https://doi.org/10.1126/science.1067524

[8] Girichidis (2016) Launching Cosmic-Ray-Driven Outflows from the Magnetized Interstellar Medium. The Astrophysical Journal Letters, 816, L19.

https://doi.org/10.3847/2041-8205/816/2/L19

[9] Mac Low, M.-M., Smith, M.D., Klessen, R.S. and Burkert, A. (1998) The Decay of Supersonic and Super-Alfvénic Turbulence in Star-Forming Clouds. Astrophysics and Space Science, 261, 195-196. https://doi.org/10.1023/A:1002036113496

[10] Mac Low, M.-M. and Klessen, R.S. (2004) Control of Star Formation by Supersonic Turbulence. Reviews of Modern Physics, 76, 125-194. https://doi.org/10.1103/RevModPhys.76.125

[11] Ballesteros-Paredes, J., Gazol, A., Kim, J., Klessen, R.S., Jappsen, A.-K., et al. (2006) The Mass Spectra of Cores in Turbulent Molecular Clouds and Implications for the Initial Mass Function. The Astrophysical Journal, 637, 384-391. https://doi.org/10.1086/498228

[12] Whitworth, A. (1979) The Erosion and Dispersal of Massive Molecular Clouds by Young Stars. Monthly Notices of the Royal Astronomical Society, 186, 59-67. https://doi.org/10.1093/mnras/186.1.59

[13] Franco, J., Shore, S.N. and Tenorio-Tagle, G. (1994) On the Massive Star-Forming Capacity of Molecular Clouds. Astrophysical Journal, 436, 795. https://doi.org/10.1086/174955

[14] Walch, S., Whitworth, A.P., Bisbas, T.G., Wünsch, R. and Hubber, D.A. (2013) Clumps and Triggered Star Formation in Ionized Molecular Clouds. Monthly Notices of the Royal Astronomical Society, 435, 917-927. https://doi.org/10.1093/mnras/stt1115

[15] Elmegreen, B.G. and Lada, C.J. (1977) Sequential Formation of Subgroups in OB Associations. Astrophysical Journal, 214, 725-741. https://doi.org/10.1086/155302

[16] Hosokawa, T. and Inutsuka, S.I. (2006) Dynamical Expansion of Ionization and Dissociation Front around a Massive Star: A Starburst Mechanism. Astrophysical Journal, 648, L131. https://doi.org/10.1086/507887

[17] Fukui, Y., et al. (2014) Molecular Clouds toward the Super Star Cluster NGC 3603; Possible Evidence for a Cloud-Cloud Collision in Triggering the Cluster Formation. 
Astrophysical Journal, 780, 36.

[18] Kumssa, G.M. and Tessema, S.B. (2019) Low Mass to Intermediate-Mass Star-Forming Hydrostatic Cores in Self-Gravitating Molecular Cloud. Astronomische Nachrichten, 340, 705-793. https://doi.org/10.1002/asna.201913543 